\title{
Effect and mechanism of RNAi targeting WWTR1 on biological activity of gastric cancer cells SGC7901
}

\author{
YUAN LI, FANG-LIANG YANG, CHUN-FU ZHU and LI-MING TANG \\ Department of General Surgery, Nanjing Medical University Affiliated Changzhou No. 2 Hospital, \\ Changzhou, Jiangsu 213164, P.R. China
}

Received April 5, 2017; Accepted September 25, 2017

DOI: $10.3892 / \mathrm{mmr} .2017 .8192$

\begin{abstract}
Gastric cancer (GC) is one of the most common malignancies in the world. It is essential to develop novel targets and therapeutic approaches for $\mathrm{GC}$, which requires identification of novel functional molecules. WW-domain containing transcription regulator 1 (WWTR1) may activate many transcriptional factors and exhibit an important role in the development of various tissues in mammals. The results of the present study demonstrated that mRNA and protein levels of WWTR1 are increased in GC tissues and cell lines. The SGC7901 cell line was selected to perform RNA interference (RNAi) targeting WWTR1, and for subsequent study. Compared with control groups (cells without any treatment) and mock groups (cells treated with nonspecific siRNA), cell proliferation of siWWTR1 cells (cells treated with WWTR1 siRNA) was detected using a Cell Counting Kit-8 assay at 12,24 and $48 \mathrm{~h}$, and decreased in a time-dependent manner. Cell cycle and apoptosis status were determined by flow cytometry, and it was demonstrated that G1/S transition was blocked in the cell cycle and apoptosis promoted in siWWTR1 cells, compared with control and mock cells. Reverse transcription-quantitative polymerase chain reaction and western blotting were performed to detect the mRNA and protein levels of cell cycle and apoptosis-associated factors. The expression of Cyclin D1, cancer Myc and B cell lymphoma/leukemia-2 (Bcl-2) decreased and Bcl-2 associated $\mathrm{X}$ protein increased significantly in siWWRT1 cells, at the mRNA and protein level, compared with control and mock cells. With the exception of the Hippo pathway, siWWTR1 regulated downstream factors, including mothers against decapentaplegic homolog family member 3 (SMAD3) and inhibitor of DNA binding 1, HLH protein (ID1), HLH protein in the transforming growth
\end{abstract}

Correspondence to: Dr Li-Ming Tang, Department of General Surgery, Nanjing Medical University Affiliated Changzhou No. 2 Hospital, 68 Gehu Mid Road, Wujin, Changzhou, Jiangsu 213164, P.R. China

E-mail:erxz7855@126.com

Key words: RNAi, WWTR1, biological activity, apoptosis, gastric cancer factor (TGF)- $\beta$ pathway. The expression of asparagine synthetase was decreased whereas ID1, SMAD3 (proteins that participate in intracellular TGF- $\beta$ transduction) and betacellulin increased notably in siWWRT1 cells. In conclusion, WWTR1 promotes cell proliferation and inhibits apoptosis of GC cells by regulating cell cycle/apoptosis-associated factors, and effectors in the TGF- $\beta$ pathway.

\section{Introduction}

Gastric cancer (GC) is a main prevalent malignancy in the world. It is considered the second most widely diagnosed malignancy causing $12 \%$ of cancer-associated deaths worldwide every year (1). The cancer incidence is extremely high in eastern Asia countries, such as China, Japan and South Korea. (2). GC mortality is on the top of the list of malignancies in China. Most of GC patients are diagnosed at late stages that missing the best chance to recover, due to the poor diagnosis in early stage (3). The metastasis ratio is more than $50 \%$, which causes difficulty to cure it. The comprehensive treatment, giving priority to chemotherapy is the main therapeutic schedule for the late stage GC. But the effect is not so good that it calls for better treatments relying on the deeper understanding of the cancer's molecular mechanism. As tumor proliferation and inhibited apoptosis are both very important reasons for malignant progression, it is essential and possible to discover new therapeutic methods and related functional molecules.

The Hippo pathway plays an important role in organism development, and its dysregulation can result in tumorigenesis. WW-domain containing transcription regulator 1 (WWTR1) is also called TAZ, transcriptional co-activator with PDZ binding motif. It acts downstream of the Hippo pathway as a transcription co-activator (4). WWTR1 can activate transcriptional factors and play an important role in tissue development. WWTR1 is also regarded as a cancer-related gene and increased WWTR1 protein levels have been found in many cancers (5). Previous studies have demonstrated that higher WWTR1 mRNA/protein levels were associated with the onset of gastric, lung, colorectal and breast cancers. The knockdown of WWTR1 in colorectal cells was reported to inhibit cell proliferation by blocking cell cycle or promoting the apoptosis (6-8). Wang et al (9) verified that WWTR1 promoted non-small cell lung cancer cell growth and inhibited 
apoptosis by Cyclin $\mathrm{A}$ and $\mathrm{C}$ transforming growth factor (TGF) regulation. Recent research indicated that high expression of WWTR1 existed in GC tissue, related to tumor TNM staging and lymph node transferance (10).

WWTR1 interacted with other regulation factors except being regulated by Hippo pathway. TGF- $\beta$ signal pathway, important in cell growth and development, has a close relationship with tumor development and progression. It inhibites tumor occuring in physiological status, but promotes tumor invasion and metastasis in tumor development process. Attisano and Wrana reported that, WWTR1 interacted with Smads in TGF- $\beta$ pathway, and Hippo activation prevented Smad nuclear accumulation and transcriptional activity (11).

Function of WWTR1 on the downstrean regulation pathway like TGF- $\beta$ pathway in GC is of significant value for GC treatment.

Although previous studies have shown that WWTR1 functioned as an oncogene in GC, the mechanism of WWTR1 modulating GC cell proliferation and apoptosis is still unclear. In order to illuminate the mechanism, we detected WWTR1 expression in GC tissue and cell lines. We examined the silencing role of siWWTR1 on the cell cycle progression and apoptosis of GC cell SGC7901 which expressed WWTR1 notably, and explored the potential mechanism to provide new thoughts for the treatment of GC.

\section{Patients and methods}

Patients and tissue samples. Informed consent was obtained before the study. A total of 52 patients aged from 38-75 years old (median age, 58 years old) with GC admitted to Hospital were enrolled, consisting of 36 males and 16 females. Among all patients there were 42 cases of gastric adenomas, and the pathological grades were: 13 cases of high differentiation, and 39 cases of low/media differentiation, while the pathological stages were: 15 cases of I+II stage, and 37 cases of III+IV stages. While 38 cases with lymphatic metastasis and 14 cases without lymphatic metastasis. No patient had received radiotherapy or chemotherapy prior to surgery. Matched adjacent normal gastric tissues were collected as negative controls Preoperative clinical and pathological follow-up data were completed by all patients. All tissue-samples of patients were collected according to the procedures approved by the institutional review board of the independent Ethics Committee, Hospital.

Cell culture. Human gastric mucosa epithelia cell GES1 and GC cell lines (SGC7901, BGC823, HGC-27, MGC-803, MKN45) purchased from ATCC (Manassas, VA, USA) were cultured in RPMI 1640 medium (Gibco, Grand Island, NY, USA) containing $10 \%$ fetal bovine serum (FBS; Gibco) with $1 \%$ penicillin/streptomycin (Invitrogen, Carlsbad, CA, USA) at $37^{\circ} \mathrm{C}, 5 \% \mathrm{CO}_{2}$ atmosphere. Cells of logarithm phase were used in our research.

siRNA transfection. siRNA-WWTR1 was designed and synthesized by GenePharma (Shanghai, China). siRNA sequence, GGTACTTCCTCAATCACAT; and control sequence, TTC TCCGAACGTGTCACGT. GC cell SGC7901 was selected for transfection and the follow-up experiments due to high-expression WWTR1. For siRNA transfection, cells were seeded onto 12-well cell culture plates at an initial density of $6 \times 10^{4}$ cells/well. When cells were $70 \%$ confluent, they were transfected with the WWTR1 siRNA and nonspecific siRNA (forming mock groups as negative control) according to the manufacturer's protocol (Qiagen, Chatsworth, CA, USA). Reverse transcription-quantitative polymerase chain reaction (RT-qPCR) and western blotting were used to measure the interference efficiency after $48 \mathrm{~h}$ transfection.

Cell viability assay. The effect of siRNA-WWTR1 on SGC7901 cell viability was detected by Cell Counting Kit-8 (CCK-8) assay. Briefly, following 12, 24, $48 \mathrm{~h}$ transfection, the cells were seeded in 96-well plates at a density of $5 \times 10^{3}$ cells/well and incubated for the indicated times. CCK- 8 $(20 \mu \mathrm{l})$ was then added to each well of the plate. Then the plate was incubated for another $1 \mathrm{~h}$. The optical density (OD) values were read at $450 \mathrm{~nm}$ by a microplate reader (Thermo Fisher Scientific, Inc., Waltham, MA, USA). Data were presented as relative proportion of viable cells to control cells.

Cell cycle analysis. Cell cycle was evaluated by propidium iodide (PI) staining after siRNA-WWTR1 transfection. Cells were trypsinized, washed twice using phosphate-buffered saline (PBS) and fixed overnight at $4^{\circ} \mathrm{C}$ in ice-cold $70 \%$ ethanol. After being washed twice with PBS, cells were incubated in $50 \mu \mathrm{g} / \mathrm{ml} \mathrm{PI}$ and $100 \mu \mathrm{g} / \mathrm{ml}$ RNAase for $30 \mathrm{~min}$ at room temperature. Thereafter, analysis was immediately performed with a FACS flow cytometer (BD Pharmingen, San Diego, CA, USA). The proportion of cells in $\mathrm{G}_{0} / \mathrm{G}_{1}, \mathrm{~S}$ and $\mathrm{G}_{2} / \mathrm{M}$ phases was detected.

Apoptosis detection. The apoptosis status was measured by Annexin V/PI double-stain assay, according to the manufacturer's protocol (BioVision, Mountain View, CA, USA). Briefly, after the interfection of siRNA-WWTR1, cells were collected and resuspended by $500 \mu \mathrm{l}$ binding buffer including $5 \mu \mathrm{l}$ Annexin V-fluorescein isothiocyanate and $5 \mu \mathrm{l}$ PI. After the incubation for $5 \mathrm{~min}$ in the dark at room temperature, cells were analyzed by a flow cytometer (BD Pharmingen). Cell Quest software was used to analyze the apoptosis rate.

\section{Independent experiments}

$R T-q P C R$. Total RNA was extracted from transfected cells, mock cells and non-transfected cells respectively and reversely transcribed to cDNA with a first strand cDNA kit (SigmaAldrich, Munich, Germany), according to the protocol provided by the manufacturer. PCR amplification was performed for $30 \mathrm{sec}$ at $95^{\circ} \mathrm{C}$, followed by 40 cycles: Denaturation at $95^{\circ} \mathrm{C}$ for $5 \mathrm{sec}$, annealing/extension at $60^{\circ} \mathrm{C}$ for $30 \mathrm{sec}$ in $\mathrm{ABI} 7300$ Thermocycler (Applied Biosystems, Foster City, CA, USA) using the SYBR Premix Ex Taq kit (Takara, Dalian, China). The primer sequences were displayed in Table I.

Western blot analysis. The concentrations of proteins were determined by BCA assay (Beyotime, Nantong, China). Then proteins were subjected to sodium dodecyl sulfate polyacrylamide gel electrophoresis (SDS-PAGE) and electroblotted onto a polyvinylidene fluoride membrane (PVDF; Amersham Pharmacia Biotech, Amersham, UK). 
Table I. Primers used in RT-PCR analysis.

\begin{tabular}{ll}
\hline Name & \multicolumn{1}{c}{ Sequence (5'-3') } \\
\hline $\begin{array}{l}\text { WWTR1 } \\
\text { Forward } \\
\text { Reverse } \\
\text { Cyclin A }\end{array}$ & CAGCCAAATCTCGTGATGAATC \\
Forward & GGTCTGCTGGCTCAGGGT \\
Reverse & GCAGAGGCCGAAGACGAGA \\
Cyclin D1 & TCCAAGGAGGAACGGTGACA \\
Forward & \\
Reverse & GCTGGAGGTCTGCGAGGA \\
Cyclin E & ACAGGAAGCGGTCCAGGTAGT \\
Forward & \\
Reverse & AGCCAGCCTTGGGACAATAAT \\
c-Myc & GAGCCTCTGGATGGTGCAAT \\
Forward & \\
Reverse & GCCACGTCTCCACACATCAG
\end{tabular}

Bcl-2

Forward

ACGGTGGTGGAGGAGCTCTT

Reverse CGGTTGACGCTCTCCACAC

XIAP

Forward

GGCACGAGCAGGGTTTCTT

Reverse

Bax

Forward
Reverse

TCCAACTGCTGAGTCTCCATATTG

ASNS

Forward
Reverse

ID1

$\begin{array}{cl}\begin{array}{c}\text { Forward } \\ \text { Reverse }\end{array} & \text { AGAGACTTTAGGGGGTGGGA } \\ \text { TMAD3 } & \text { TGAGAAGCACCAAACGTGAC } \\ \text { Forward } & \text { TTCACTGGTGCTGGGGTTAG } \\ \text { Reverse } & \text { GGCGGCAGTAGATGACATGA } \\ \text { BTC } & \\ \text { Forward } & \text { GAAATGGAAACTCTGGGT } \\ \text { Reverse } & \text { CAAATGAGCAAGGCACT } \\ \text { GAPDH } & \\ \text { Forward } & \text { TGACTTCAACAGCGACACCCA } \\ \text { Reverse } & \text { CACCCTGTTGCTGTAGCCAAA }\end{array}$

WWTR1, WW-domain containing transcription regulator 1; c-Myc, cancer Myc; Bcl-2, B cell lymphoma/leukemia-2; XIAP, X-linked inhibitor of apoptosis; Bax, Bcl-2 associated $\mathrm{X}$ protein; ASNS, asparagine synthetase (glutamine-hydrolyzing); ID1, inhibitor of DNA binding 1, HLH protein; SMAD3, mothers against decapentaplegic homolog family member 3; BTC, betacellulin; GAPDH, glyceraldehyde-3-phosphate dehydrogenase.

Following blockage with 5\% non-fat dry milk in PBS for $1 \mathrm{~h}$, the blotting membranes were probed overnight respectively at $4^{\circ} \mathrm{C}$, with the following primary antibodies: Rabbit anti-WWTR1 (ab84927, 1:500), mouse anti-Cyclin D1 (ab6152, 1:1,000), mouse anti-cancer Myc (c-Myc; ab17356, 1:1,000), mouse anti-B cell lymphoma/leukemia-2 (Bcl-2; ab692, 1:1,000), mouse anti-Bcl-2 associated X protein (Bax; ab77566, 1:1,000); mouse anti-asparagine synthetase (ASNS; ab171800, 1:1,000), mouse anti-inhibitor of DNA binding 1, HLH protein (ID1; ab168256, 1:1,000), mouse anti-mothers against decapentaplegic homolog family member 3 (SMAD3; ab55480, 1:500), mouse anti-betacellulin (BTC; ab89156, 1:500), and mouse anti-glyceraldehyde-3-phosphate dehydrogenase (GAPDH; ab9484, 1:5,000; all from Abcam, Cambridge, UK), then they were probed with the appropriate HRP-conjugated secondary antibodies: rabbit anti mouse IgG $\mathrm{H}+\mathrm{L}$ (HRP) (ab6728, 1:5,000), goat anti rabbit IgG H+L (HRP) (ab6721, 1:5,000; both from Abcam). The PVDF membrane was exposed to X-ray film and immunoreactive bands were detected by reaction with Enhanced Chemiluminescense Detection system reagents (Amersham, Arlington Heights, IL, USA). For loading control, the membrane was probed with a monoclonal antibody for GAPDH. Lab Works Image Acquisition and Analysis Software (UVP, Inc., Upland,. CA, USA) were used to quantify band intensities.

Statistical analysis. All results were presented as mean \pm SD of three independent experiments. Statistical analysis was performed using a SPSS 13.0 statistical package and data were subjected to one-way analysis of variance, followed by Dunnett's test. $\mathrm{P}<0.05$ was considered significant, $\mathrm{P}<0.01$ was considered especially significant.

\section{Results}

High expression of WWTRl in GC tissue. To verify the biological role of WWTR1 in GC, the expression levels of WWTR1 in GC tissues were detected by RT-PCR and western blotting. It showed that the mRNA expression of WWTR1 was much higher in GC tissues, and the protein expression of WWTR1 was much higher in most of GC tissues than that of adjacent normal tissues $(\mathrm{P}<0.01)$, we choose some representative blots to diaplay in Fig. 1. It indicated that the over-expression of WWTR1 may be involved in the initiation and/or progression of GC (Fig. 1A-C).

WWTR1 expression in GC cell lines. Based on the evident difference in WWTR1 expression between GC tissues and normal tissues, the mRNA and protein expression of WWTR1 in normal gastric mucosa epithelia cell GES1 and GC cell lines including SGC7901, BGC823, HGC-27 MGC-803 and MKN45 were detected by RT-PCR and western blotting. The mRNA expression of WWTR1 was remarkably higher in SGC7901 cell than in any other cell lines, twice of GES1 cells (Fig. 1D). And the protein level of WWTR1 in SGC7901 was the second highest among all cell lines. Protein level of WWTR1 in BGC823 was a little higher than that of SGC7901, but of no significant difference. And both mRNA and protein levels of SGC7901 increased remarkably compared with GES1 (control group) (Fig. 1E and F). While SGC7901 is used in more study, of representative meaning, it was chosen to be used in our study. 
A

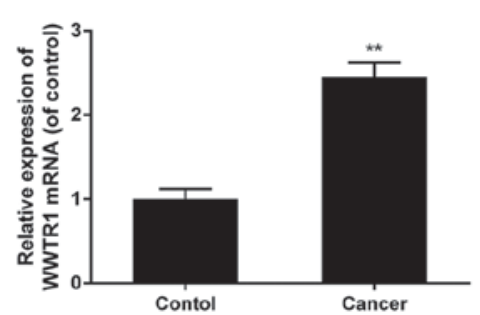

D

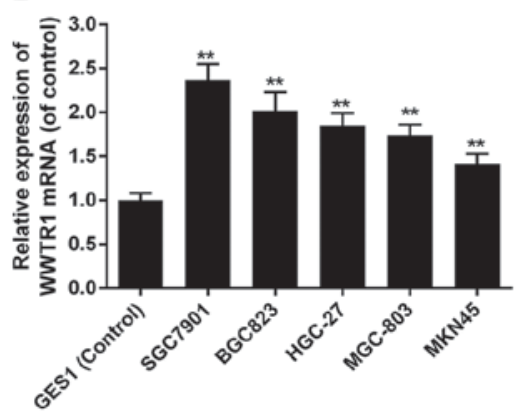

B

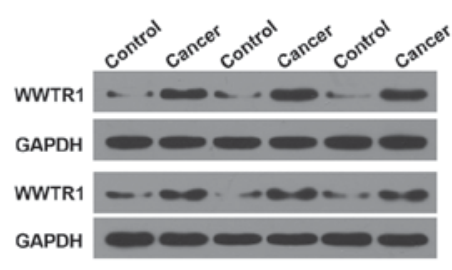

E

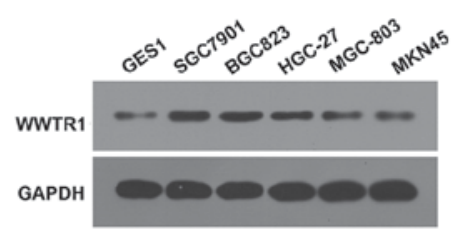

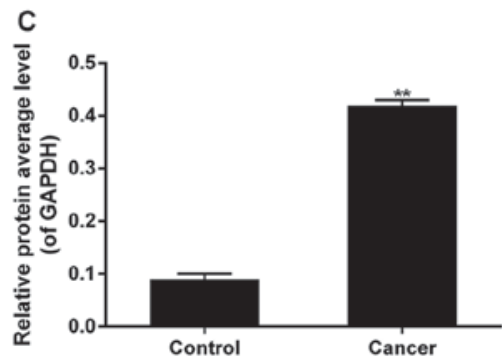

$\mathrm{F}$

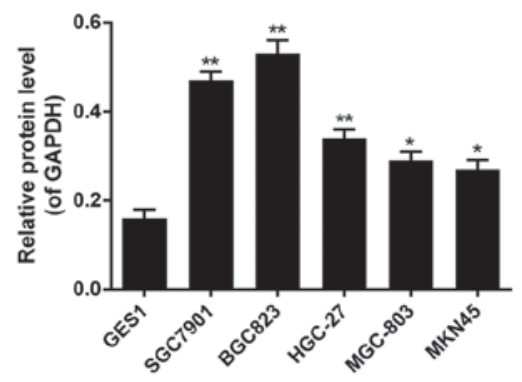

Figure 1. WWTR1 expression in gastric cancer tissue and cell lines. (A) WWTR1 mRNA expression was measured by RT-PCR in gastric cancer tissue and the adjacent normal tissue. (B and C) Protein levels of WWTR1 were determined by western blotting in gastric cancer tissue and adjacent normal tissue. Several representative western blotting images were presented. The average protein level was presented in (C). (D) WWTR1 mRNA expression was measured by RT-PCR in normal gastric mucosa epithelia cell GES1 and gastric cancer cell lines including SGC7901, BGC823, HGC-27 MGC-803 and MKN45. (E and F) Protein levels of WWTR1 were determined by western blotting in cell lines (GES1, SGC7901, BGC823, HGC-27 MGC-803 and MKN45). GAPDH was detected as the control of sample loading. Data were presented as mean $\pm \mathrm{SD}, \mathrm{n}=6$; ${ }^{\mathrm{P}} \mathrm{P}<0.05$ and ${ }^{* *} \mathrm{P}<0.01$ compared with the control cells. WWTR1, WW-domain containing transcription regulator 1; GAPDH, glyceraldehyde-3-phosphate dehydrogenase.

A

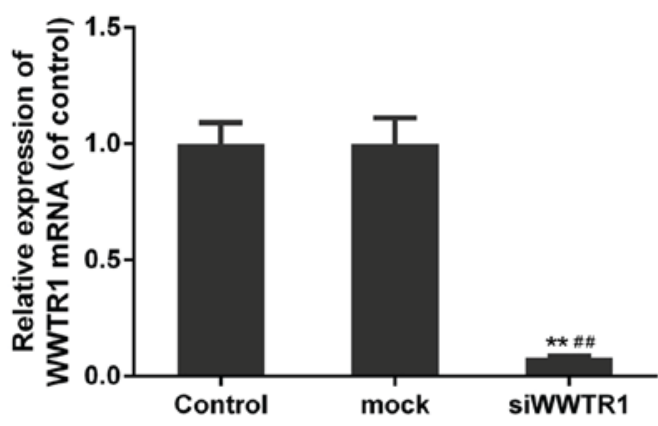

C

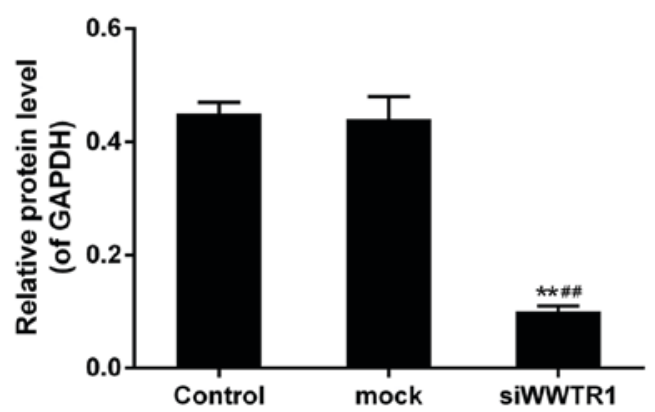

B
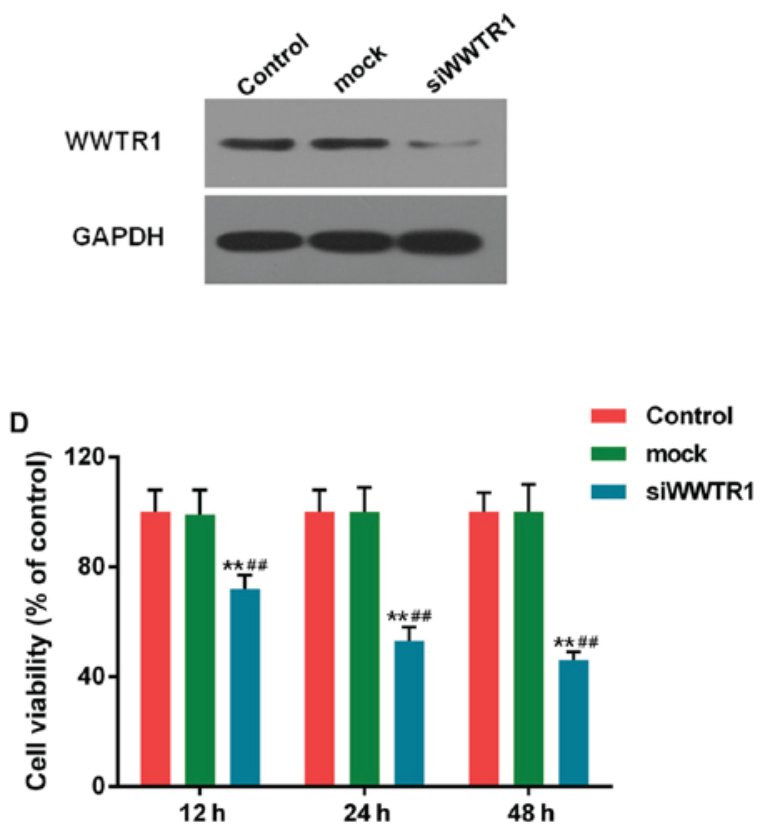

Figure 2. Effect of siRNA-WWTR1 on cell viability of SGC7901 cell. (A) After siRNA-WWTR1 transfection for 48 h, mRNA expression of WWTR1 in SGC7901 cells was quantified by RT-PCR. (B and C) Protein levels of WWTR1 in SGC7901 cells were measured by western blotting after siRNA-WWTR1 transfection for $48 \mathrm{~h}$. (D) After siRNA-WWTR1 transfection for 12, 24 and $48 \mathrm{~h}$, CCK-8 assay was performed to verify the cell viability of SGC7901 cells. Data were presented as mean $\pm \mathrm{SD}, \mathrm{n}=6 .{ }^{* *} \mathrm{P}<0.01$ compared with the control cells and ${ }^{\# \#} \mathrm{P}<0.01$ compared with the mock cells. WWTR1, WW-domain containing transcription regulator 1; CCK-8, Cell Counting Kit-8; GAPDH, glyceraldehyde-3-phosphate dehydrogenase.

Effect of siRNA-WWTR1 on SGC7901 cell viability. WWTR1 mRNA was interfered in SGC7901 cell line as previously described. The interference efficience was then identified by means of RT-PCR and western blotting. As displayed in (Fig. 2A-C), transfection of siRNA-WWTR1 resulted in dramatic decline in WWTR1 mRNA and protein levels in 
A
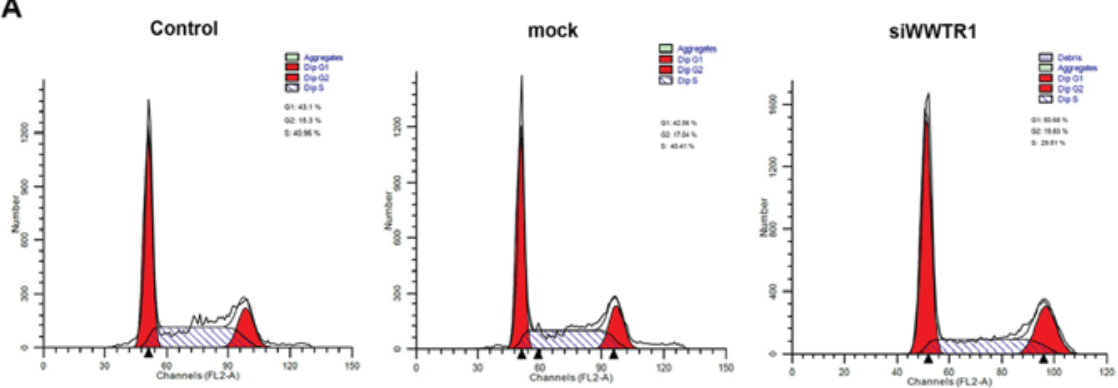

C
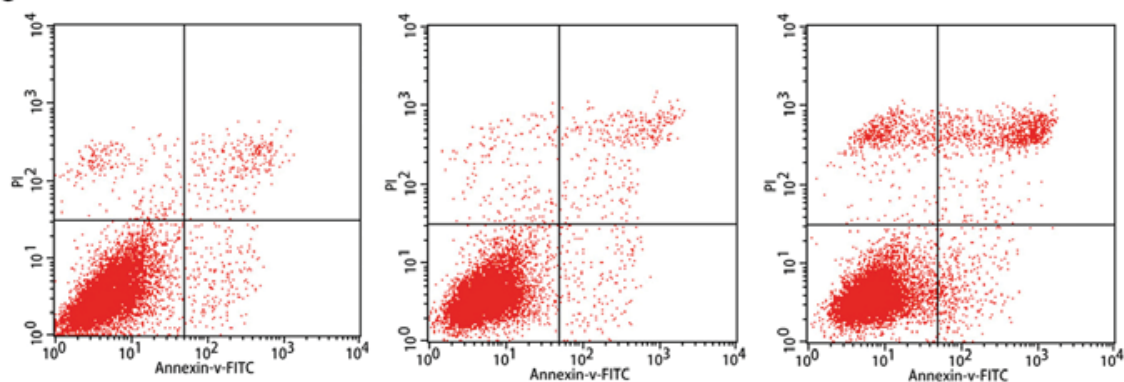

B

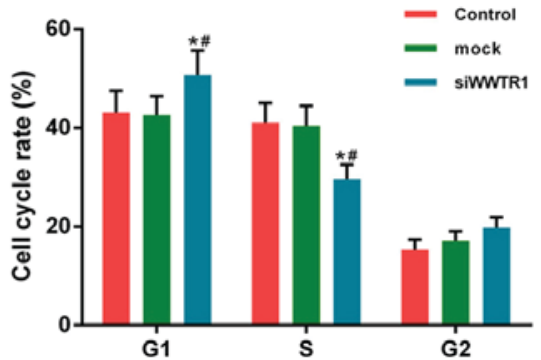

D

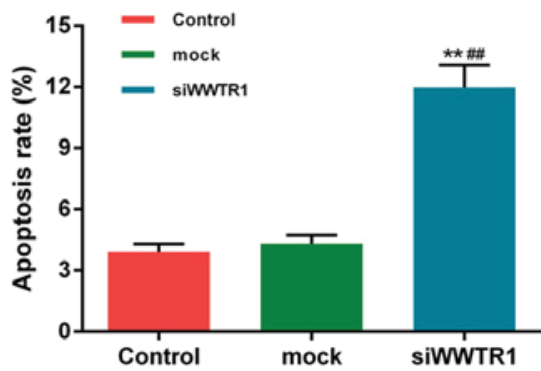

Figure 3. Effect of siRNA-WWTR1 on cell cycle progression and apoptosis of SGC7901 cell. (A and B) At siRNA-WWTR1 48 h transfection, cell cycle analysis of SGC7901 was determined, checking the proportion of cells in G1, S and G2 phases respectively. (C and D) At siRNA-WWTR1 48 h transfection, cell apoptosis of SGC7901 was detected by Annexin V/PI double-stain analysis. Data were presented as mean $\pm \mathrm{SD}, \mathrm{n}=6$. * $\mathrm{P}<0.05$ and ${ }^{* *} \mathrm{P}<0.01 \mathrm{compared}$ with the control cells, ${ }^{\#} \mathrm{P}<0.05$ and ${ }^{\# \#} \mathrm{P}<0.01$ compared with the mock cells. WWTR1, WW-domain containing transcription regulator 1 ; PI, propidium iodide.

siRNA-WWTR1 group compared with the control group and mock group, which confirmed that siRNA-WWTR1 was effective in silencing WWTR1 expression.

The effect of siRNA-WWTR1 on SGC7901 cell viability measured by CCK-8 assay was shown in Fig. 2D. The cell viability was significantly suppressed in siRNA-WWTR1 group 24 and $48 \mathrm{~h}$ post transfection compared with that of control and mock cells.

Effect of siRNA-WWTR1 on SGC7901 cell cycle arrest and apoptosis. As we can see, cell viability was decreased in siRNA-WWTR1 group, we examined the effect of WWTR1 on cell cycle progression and apoptosis. As displayed in (Fig. 3A and B), pretreatment of GC cells with siRNA-WWTR1 blocked the cell cycle transition from $\mathrm{G} 1$ to $\mathrm{S}$ phase. The percentage of cells in G0/G1-phases increased from 43.10 to $50.68 \%$, while S-phase fraction decreased from 40.96 to $29.51 \%$.

We performed Annexin V/PI double-staining to quantify apoptotic cell death, and used flow cytometry for analysis (Fig. 3C and D). At $48 \mathrm{~h}$ after transfection with siWWTR1, apoptosis increased as the phosphatidylserine (PS) extrution to the outer leaflet of the plasma membrane increased, which was detected by Annexin V binding to the surface of cells. The apoptosis of SGC7901 cell increased 3-fold, compared with control and mock groups.

Effect of siRNA-WWTR1 on cell cycle and apoptosis related factors in SGC7901. The critical role of siRNA-WWTR1 in SGC7901 cell cycle blocking and induction of apoptosis promoted us to further explore the potential mechanism by measuring the relevant genes and proteins. The RNA levels of Cyclin D1, c-Myc, Bcl-2 were decreased dramatically, and Bax increased significantly, while Cyclin A, Cyclin E and X-linked inhibitor of apoptosis (XIAP) changed little in siWWRT1-interferring cells compared to the control and mock cells (Fig. 4A). Western blotting was performed to measure the protein expression of Cyclin D1, c-Myc, Bcl-2 and Bax, for the mRNA expression of them changed significantly. The protein level of Cyclin D1, c-Myc, Bcl-2 were decreased and Bax increased significantly in siWWRT1-interferring cells compared with control and mock cells, being consistent with mRNA variation (Fig. 4B and C). The results indicated regulation of WWTR1 on cell proliferation and apoptosis may rely on the expression of Cyclin D1, c-Myc, Bcl-2 and Bax.

Effect of siRNA-WWTR1 on WWTR1 downstream effectors in $S G C 7901$. Regardless of Hippo pathway, we explored the role of WWTR1 on the downstream effectors in other signaling pathways like TGF- $\beta /$ Smad pathway and so on. RT-PCR and western blotting were performed to assess the expression of WWTR1 downstream factors such as ASNS, ID1, SMAD3 and BTC, reported important in tumors. The expression of ASNS was decreased notably, while ID1, SMAD3 and BTC increased significantly in siWWRT1-interferring cells compared with control and mock cells, both in RNA and protein manners (Fig. 5).

\section{Discussion}

Being the second widely diagnosed malignancy in the world, most of GCs are diagnosed at late stage due to the poor diagnosis in early stage. The development and metastasis of tumor is a multi-step process including lots of genetic variations. Apoptosis is a process of programmed cell death that occurs in multicellular organisms (12). Decreased apoptosis causes uncontrolled cell proliferation, such as tumor (13). As 
A

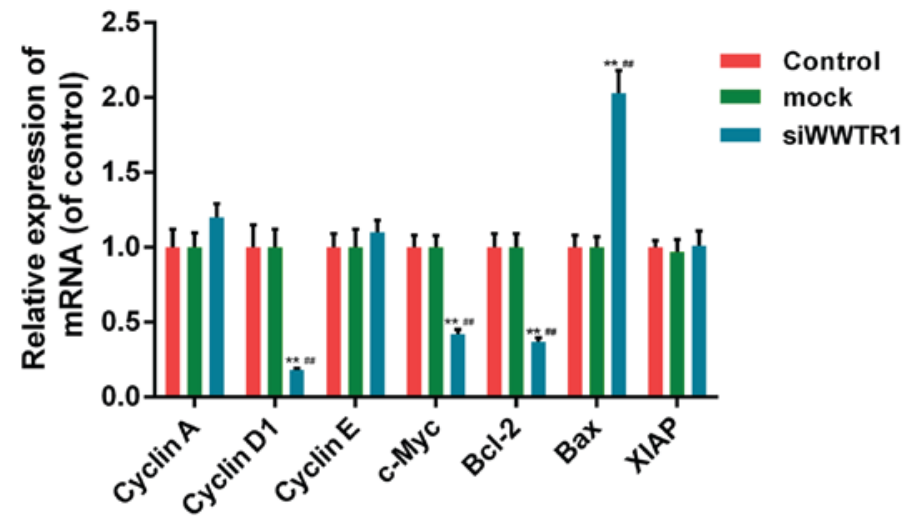

B

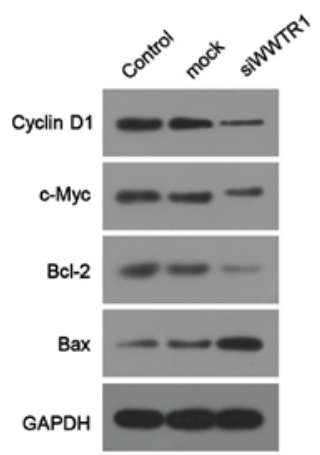

C

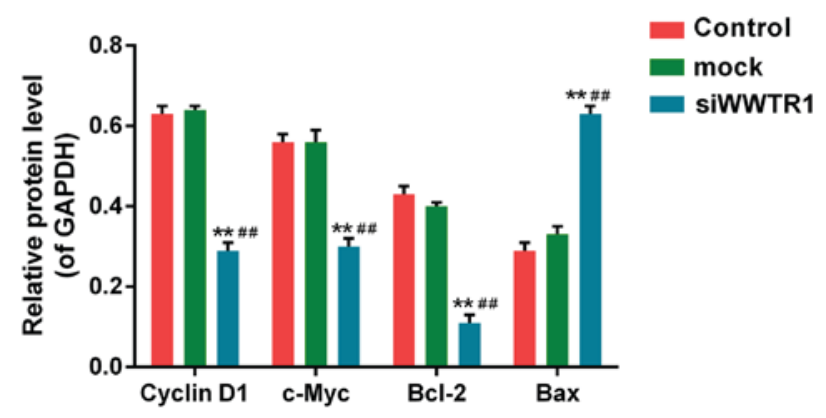

Figure 4. Effect of siRNA-WWTR1 on expression of cell cycle and apoptosis related factors. (A) RT-PCR was conducted for mRNA expression detection of cell cycle factors such as Cyclin A, Cyclin D1, Cyclin E, c-Myc, and apoptosis related factors such as Bcl-2, XIAP and Bax, in SGC7901 cells after siRNA-WWTR1 transfection for $48 \mathrm{~h}$. (B and C) The protein levels of Cyclin D1, c-Myc, Bcl-2 and Bax in SGC7901 cells were analyzed by western blotting after $48 \mathrm{~h}$ of siRNA-WWTR1 treatment. GAPDH was detected as the control of sample loading. Data were presented as mean $\pm \mathrm{SD}, \mathrm{n}=6,{ }^{* * *} \mathrm{P}<0.01 \mathrm{vs}$. control; ${ }^{\# \#} \mathrm{P}<0.01$ vs. mock. WWTR1, WW-domain containing transcription regulator 1; Bcl-2, B cell lymphoma/leukemia-2; XIAP, X-linked inhibitor of apoptosis; Bax, Bcl-2 associated X protein; GAPDH, glyceraldehyde-3-phosphate dehydrogenase; c-Myc, cancer Myc.

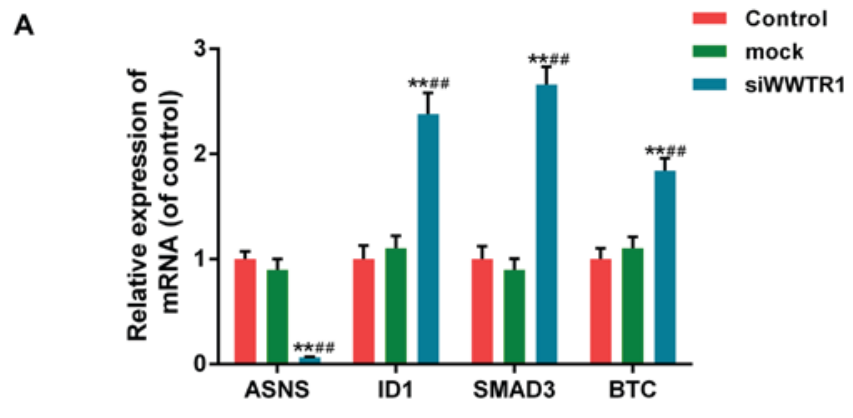

B

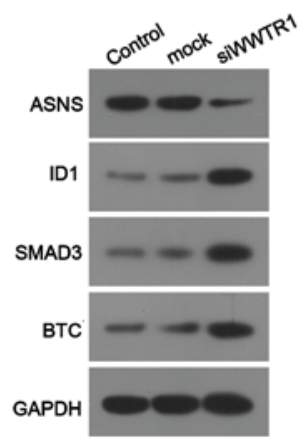

C

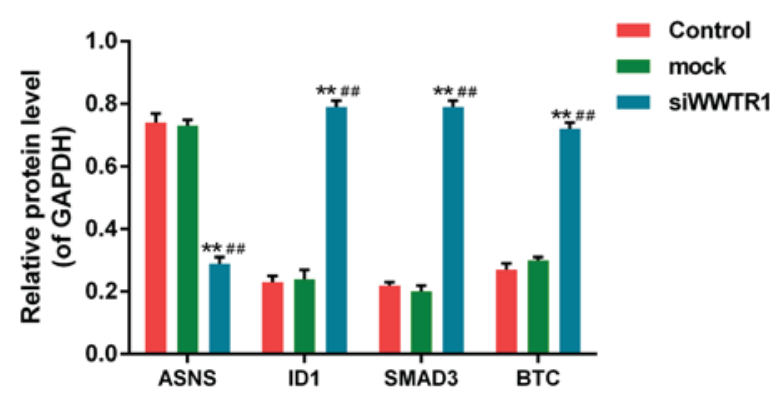

Figure 5. Effect of siRNA-WWTR1 on expression of WWTR1 downstream factors. (A) After siRNA-WWTR1 transfection for $48 \mathrm{~h}$, the mRNA expression of ASNS, ID1, SMAD3 and BTC were measured by RT-PCR. (B and C) ASNS, ID1, SMAD3 and BTC protein expression were determined by western blotting after siRNA-WWTR1 transfection for $48 \mathrm{~h}$. GAPDH was also detected as the control of sample loading. Data were presented as mean $\pm \mathrm{SD}, \mathrm{n}=6,{ }^{* *} \mathrm{P}<0.01 \mathrm{vs}$. control; ${ }^{\# \#} \mathrm{P}<0.01$ vs. mock. WWTR1, WW-domain containing transcription regulator 1; ASNS, asparagine synthetase (glutamine-hydrolyzing); ID1, inhibitor of DNA binding 1, HLH protein; SMAD3, mothers against decapentaplegic homolog family member 3; BTC, betacellulin; GAPDH, glyceraldehyde-3-phosphate dehydrogenase. 
tumor proliferation and inhibited apoptosis are main causes of malignant progression, it is essential and possible to discover new therapeutic methods and related functional molecules.

WWTR1 mainly acts downstream of the Hippo pathway as a transcription co-activator (14). It is also regarded as a cancer-related gene and increased WWTR1 protein levels have been found in some cancers (15). Although previous studies have shown that WWTR1 functioned as an oncogene in GC, the mechanism of WWTR1 modulating GC cell proliferation and apoptosis is still unclear.

In the present study, we explored that the expression of WWTR1 in most of GC tissues and cells exactly increased notably. SGC7901 was selected for further research for the significant increasing of WWTR 1 mRNA and protein compared with GES1 (control group). Certainly the study on BGC823 which presented highest protein level maybe also an interesting research project, we would keep an eye on it and investigate the molecular mechanism in it in future. For the elevated levels of WWTR1, we chose silencing as the strategy to study more. As WWTR1 levels improved not too much in some cell lines like MKN45, maybe we could also do more research on it by over-exprssion strategy next time.

As results demonstrated, siRNA-WWTR1 effectively inhibited the vitality of the cells. Then we explored the function of siWWTR1 on cell cycle progression and apoptosis, finding siWWTR1 inhibiting cell proliferation, blocking cell cycle transition from G1 to $\mathrm{S}$ phase, and promoting apoptosis significantly. We also provided evidence that the mechanism underlying the above effects was related to the adjustment of cell cycle and apoptosis-related factors.

Cyclin D1 is an important factor in cell G1 progressing (16). C-Myc encodes phosphorylated proteins, which facilitate cell proliferation and differentiation (17). Bcl-2 activates caspase pathway, facilitates cell mitosis and inhibits apoptosis by dissolving protein structures, resulting in tumor proliferation. On the contrary (18), Bax can facilitate apoptosis by preventing function of Bcl-2 as forming heterodimer with it. Our research found that siWWTR1 suppressed expression of Cyclin D1, c-Myc, Bcl-2 and increased expression of Bax, which could lead to interference of cell proliferation, cell cycle blocking and apoptosis promoting. Wang et al (9) found that Cyclin A and connect tissure growth factor (CTGF) levels were inhibited by siWWTR1 in non-small cell lung cancer cells, but the levels of Cyclin D1, Cyclin E, c-Myc, Bcl-2, Bax and XIAP did not change too much. Though a little different with our results, it is reasonable for different diseases.

Regardless of Hippo pathway, we explored the role of WWTR1 on the downstream effectors in other signaling pathways like TGF- $\beta /$ Smad and so on in SGC7901. The family of TGF- $\beta$ can adjust cell proliferation or differentiation, apoptosis and migration. The biological function depends on the normal signaling pathway. The dysregulation of them can result in tumor initiation and development. Smad is a critical factor in TGF- $\beta$ pathway $(19,20)$, Researches before had proved the genetic defect of it can induce ubiquitylation-mediated degradation increase, related to colorectal cancer $(21,22)$. Id1 can promote cell proliferation by inhibiting the expression of P21, the inhibitor of cyclin-dependent kinase and promoting cell cycle transition from $\mathrm{G} 1$ to $\mathrm{S}$ phase $(23,24)$. In our present study, we found that the expression of ID1, SMAD3 increased remarkably in siWWTR1-interferring cells compared to the control and mock cells, both in RNA and protein manners. It indicated that WWTR1 participated in TGF- $\beta$ pathway to enhance tumor proliferation by inhibiting the expression of SMAD3 and ID1.

BTC is a-member of epidermal growth factor (EGF) family. BTC can inhibit cell apoptosis by promoting the phosphorylation of EGR receptor (EGFR) (25). EGFR is an important binding site of targeted drug for cancers. In our present study, we found that the expression of BTC increased significantly in siWWTR1-interferring cells compared to the control and mock cells, both in RNA and protein manners. The phenomenon occurred maybe due to the inhibition of EGFR phosphorylation or blocking of EGFR downstream pathway, which made BTC function impossible. Though it needs proving furthermore, the result would supply novel basis for EGFR targeting treatment.

ASNS is a member of aminotransferase family (26). ASNS is related to G1-S phase transition, promoting cell mitosis and proliferation. In our study, the expression of ASNS was decreased notably in siWWRT1-interferring cells compared to the control and mock cells in RNA and protein manners, indicating WWTR1 may positive regulate ASNS expression which related to cell proliferation and tumor development. It indicated that WWTR1 may regulate cell proliferation not only by Hippo pathway, but also related to TGF- $\beta$ pathway including SMAD3 and ID1, and factors in other pathways like ASNS and BTC.

In conclusion, our study showed that WWTR 1 was highly expressed in human GC tissues/cells. Furthermore, siRNA-WWTR1 can notably inhibit cell proliferation, block cell cycle, and promote apoptosis of SGC7901 cells by regulating expression of cycle and apoptosis-related factors such as Cyclin D1, c-Myc, Bcl-2 and Bax. And the role of WWTR1 could be related to TGF- $\beta$ pathway including SMAD3 and ID1, and factors in other pathways like ASNS and BTC. It may provide novel effective therapeutic strategy for GC.

\section{References}

1. Hamashima C: Current issues and future perspectives of gastric cancer screening. World J Gastroentero 20: 13767-13774, 2014.

2. Hamashima C, Sasazuki S, Inoue M and Tsugane S: JPHC Study Group: Receiver operating characteristic analysis of prediction for gastric cancer development using serum pepsinogen and Helicobacter pylori antibody tests. BMC Cancer 17: 183, 2017.

3. Bang YJ, Van Cutsem E, Feyereislova A, Chung HC, Shen L, Sawaki A, Lordick F, Ohtsu A, Omuro Y, Satoh T, et al: Trastuzumab in combination with chemotherapy versus chemotherapy alone for treatment of HER2-positive advanced gastric or gastro-oesophageal junction cancer (ToGA): A phase 3 , open-label, randomised controlled trial. Lancet 376: 687-697, 2010.

4. Yang S, Ji M, Zhang L, Chen Y, Wennmann DO, Kremerskothen J and Dong J: Phosphorylation of KIBRA by the extracellular signal-regulated kinase (ERK)-ribosomal S6 kinase (RSK) cascade modulates cell proliferation and migration. Cell Signal 26: 343-351, 2014.

5. Fujiwara S, Ida H, Yoshioka Y, Yoshida $H$ and Yamaguchi M: The warts gene as a novel target of the Drosophila DRE/DREF transcription pathway. Am J Cancer Res 2: 36-44, 2012.

6. Dong J, Feldmann G, Huang J, Wu S, Zhang N, Comerford SA, Gayyed MF, Anders RA, Maitra A and Pan D: Elucidation of a universal size-control mechanism in Drosophila and mammals. Cell 130: 1120-1133, 2007. 
7. Wu S, Huang J, Dong J and Pan D: Hippo encodes a Ste-20 family protein kinase that restricts cell proliferation and promotes apoptosis in conjunction with salvador and warts. Cell 114: 445-456, 2003 .

8. Zhao B, Ye X, Yu J, Li L, Li W, Li S, Yu J, Lin JD, Wang CY, Chinnaiyan AM, et al: TEAD mediates YAP-dependent gene induction and growth control. Genes Dev 22: 1962-1971, 2008.

9. Wang L, Chen Z, Wang Y, Chang D, Su L, Guo Y and Liu C: TR1 promotes cell proliferation and inhibits apoptosis through cyclin A and CTGF regulation in non-small cell lung cancer. Tumour Biol 35: 463-468, 2014.

10. Justice RW, Zilian O, Woods DF, Noll M and Bryant PJ: The Drosophila tumor suppressor gene warts encodes a homolog of human myotonic dystrophy kinase and is required for the control of cell shape and proliferation. Genes Dev 9: 534-546, 1995.

11. Attisano L and Wrana JL: Signal integration in TGF- $\beta$, WNT, and Hippo pathways. F1000Prime Rep 5: 17, 2013.

12. Böhm I and Schild H: Apoptosis: The complex scenario for a silent cell death. Mol Imaging Biol 5: 2-14, 2003.

13. Zhu M, Li W, Dong X, Chen Y, Lu Y, Lin B, Guo J and Li M: Benzyl-isothiocyanate induces apoptosis and inhibits migration and invasion of hepatocellular carcinoma cells in vitro. J Cancer 8: 240-248, 2017.

14. Zhang Y, Xue C, Cui H and Huang Z: High expression of TAZ indicates a poor prognosis in retinoblastoma. Diagn Pathol 10: 187, 2015.

15. Edgar BA: From cell structure to transcription: Hippo forges a new path. Cell 124: 267-273, 2006.

16. Wang P, Liu S, Cheng B, Wu XZ, Ding SS, Xu L, Liu Y, Duan L and Sun SZ: Promoting effect of cyclin D1 overexpression on proliferation and epithelial mesenchymal transition of cervical squamous cell carcinoma SiHa cells. Zhonghua Bing Li Xue Za Zhi 46: 187-192, 2017 (In Chinese).

17. Zhou L, Wu F, Jin W, Yan B, Chen X, He Y, Yang W, Du W, Zhang Q and Guo Y: Theabrownin inhibits cell cycle progression and tumor growth of lung carcinoma through c-myc-related mechanism. Front Pharmacol 8: 75, 2017.
18. Sims EK, Lakhter AJ, Anderson-Baucum E, Kono T, Tong X and Evans-Molina C: MicroRNA 21 targets BCL2 mRNA to increase apoptosis in rat and human beta cells. Diabetologia 60: 1057-1065, 2017.

19. Luwor RB, Hakmana D, Iaria J, Nheu TV, Simpson RJ and Zhu HJ: Single live cell TGF- $\beta$ signalling imaging: Breast cancer cell motility and migration is driven by sub-populations of cells with dynamic TGF- $\beta$-Smad3 activity. Mol Cancer 14: 50, 2015.

20. Dai F, Lin X, Chang C and Feng XH: Nuclear export of Smad2 and Smad3 by RanBP3 facilitates termination of TGF-beta signaling. Dev Cell 16: 345-357, 2009.

21. Xu J and Attisano L: Mutations in the tumor suppressors Smad2 and Smad4 inactivate transforming growth factor beta signaling by targeting Smads to the ubiquitin-proteasome pathway. Proc Natl Acad Sci USA 97: 4820-4825, 2000.

22. Petersen M, Pardali E, van der Horst G, Cheung H, van den Hoogen C, van der Pluijm G and Ten Dijke P: Smad2 and Smad3 have opposing roles in breast cancer bone metastasis by differentially affecting tumor angiogenesis. Oncogene 29: 1351-1361, 2010.

23. Yu Y, Liang Y, Yin C, Liu X, Su Y, Zhang L and Wang H: Inhibitor of DNA-binding 1 promotes endothelial progenitor cell proliferation and migration by suppressing E2-2 through the helix-loop-helix domain. Int J Mol Med 38: 1549-1557, 2016.

24. Zhao Y, Luo A, Li S, Zhang W, Chen H, Li Y, Ding F, Huang F and Liu Z: Inhibitor of differentiation/DNA binding 1 (ID1) inhibits etoposide-induced apoptosis in a c-Jun/c-Fos-dependent manner. J Biol Chem 291: 6831-6842, 2016.

25. Singh B, Carpenter G and Coffey RJ: EGF receptor ligands: Recent advances. F1000Res 5: pii: F1000 Faculty Rev-2270, 2016.

26. Xu Y, Lv F, Zhu X, Wu Y and Shen X: Loss of asparagine synthetase suppresses the growth of human lung cancer cells by arresting cell cycle at G0/G1 phase. Cancer Gene Ther 23: 287-294, 2016.

(i) (5) $\odot$ This work is licensed under a Creative Commons Attribution-NonCommercial-NoDerivatives 4.0 International (CC BY-NC-ND 4.0) License. 